\title{
Bioquímica. La importancia de las áreas básicas en la odontología
}

\author{
Biochemistry. The importance of basic sciences in dentistry
}

\author{
Silvia Maldonado-Frías*
}

Los seres vivos son un conjunto complejo de elementos químicos unidos entre sí. Las estructuras que los constituyen tienen una armoniosa disposición, la cual les permite trabajar de una forma concertada para dar lugar a un sin número de eventos celulares que mantienen una comunicación interna entre moléculas que requieren de energía y son portadoras de ella. Del mismo modo, los seres vivos reciben mensajes provenientes del entorno y los traducen de manera específica y coordinada, produciendo respuestas que permiten el desarrollo y reproducción de las distintas formas de vida.

La bioquímica es una disciplina científica que explica cómo el carbono, hidrógeno, oxígeno, nitrógeno, fósforo y azufre, además de ser imprescindibles para la vida, son componentes de estructuras como los carbohidratos, lípidos, proteínas y ácidos nucleicos, las cuales están implicadas en el metabolismo celular. La bioquímica también aporta conocimientos valiosos respecto a la compleja relación molecular que permite sustentar la vida; de la misma manera permite comprender los procesos que acompañan el envejecimiento y la muerte celular, saber acerca de la transformación de energía en los seres vivos y de los mecanismos de señalización, así como proveer de saberes necesarios para entender las investigaciones científicas y tecnológicas.

Conocer y comprender cada uno de los eventos que permiten un adecuado funcionamiento, sienta las bases para entender que la alteración de estos procesos resulta en los distintos trastornos que aquejan a los seres vivos. De ahí que la bioquímica no esté aislada y sea parte de un distinguido grupo de disciplinas que interactúan para la total comprensión del maravilloso cuerpo humano.

De sobra debe entenderse que la bioquímica, que ha sido el trampolín de un sin número de premios nobel como el otorgado a Fischer en 1902 (quien concluyó que la acción de una enzima era específica en relación con un substrato), es sin duda una disciplina que pro- vee herramientas significativas en el desarrollo de la investigación médica y sustenta su importancia dentro del plan de estudios de la carrera de cirujano dentista.

Bruce J. Baum comenta que las ciencias biomédicas son abrumadoramente relevantes e inciden en el ejercicio odontológico, y pone de manifiesto que pareciera existir resistencia para considerar su importancia en la educación dental. Señala también que apreciar y comprender la ciencia y pensar científicamente, no denigra los conocimientos técnicos requeridos en la práctica dental. Por el contrario, entender a la biología de los tejidos y de la enfermedad, utilizando el pensamiento lógico riguroso en la prestación de la atención clínica, y valorando cómo las ciencias biomédicas conducen a los avances terapéuticos, son condiciones deseables bajo distintas circunstancias para cualquier disciplina clínica, incluyendo todas las facetas de la odontología. ${ }^{1}$ Los avances científicos se presentan de forma exponencial y crean una variedad de conocimientos disponibles para la comprensión de los fenómenos que suceden en el cuerpo humano. Actualmente, se desarrollan herramientas poderosas que pueden ser aplicadas en el tratamiento de las enfermedades, como el uso de nanopartículas en implantes dentales. Sin embargo, hacer llegar estos conocimientos a las aulas para crear un impacto en la enseñanza odontológica es incierto.

lacopino señala la carencia de personal con formación adecuada y con experiencia en las ciencias básicas y clínicas que permitan la efectiva integración y aplicación de conocimientos emergentes que provee la ciencia. ${ }^{2}$ Estas carencias podrán ser suplidas cuando se reconozca el rol de las áreas básicas en la educación odontológica.

\footnotetext{
* Facultad de Odontología, Universidad Nacional Autónoma de México (UNAM).
}

Este artículo puede ser consultado en versión completa en http://www.medigraphic.com/facultadodontologiaunam 
Otros autores señalan que la formación profesional en los proveedores de la salud contempla las competencias básicas en la promoción de la salud y prevención de enfermedades, poniendo de manifiesto que es un objetivo en las escuelas de Canadá y Estados Unidos de Norteamérica; sin embargo, un punto de convergencia en estos estudios es la inclusión de las áreas básicas dentro de sus planes de estudio, e incluso han incrementado el número de cursos interdisciplinarios en estas áreas; también indican que existen disciplinas básicas que comparten en su formación académica los profesionales de la salud, y que por ello deben de crearse puentes de comunicación entre los proveedores de la salud para ofrecer una atención de calidad. ${ }^{3-6}$

El Dr. Zoller propone la deconstrucción del currículum en la enseñanza odontológica, remarcando que existen contenidos que no atañen al odontólogo, a lo que el Dr. Zallen responde que el recorte en el plan de estudios de las disciplinas básicas podría originar la formación de un dentista que no entendiera la complejidad de las enfermedades y los procesos quirúrgicos con los cuales su paciente puede presentarse, poniéndolos en riesgo cuando estos busquen atención dental. ${ }^{7,8}$

En una sesión sobre la renovación de la educación dental en Estados Unidos de Norteamérica se recopilaron las aportaciones y conocimientos generados en la misma. Es destacado comentar la intervención del Dr. Tabak. Él plantea los siguientes cuestionamientos: ¿los dentistas deberán ser educados en el más alto nivel como los oftalmólogos o en un nivel más técnico como los optometristas?, ¿cómo se forman los dentistas en términos de experiencia y saber?, ¿van a comprender plenamente la biología de la salud oral y de la enfermedad y ser capaces de tratar las condiciones más complejas? Concluyendo que las escuelas tienen que construir su base de conocimientos científicos, así como impulsar la dedicación a la investigación científica y el conocimiento entre los estudiantes de odontología. Turnes por su parte señaló en la misma sesión que la odontología está correctamente enfocada en la necesidad de transformar el contenido y la metodología de enseñanza a fin de mejorar su pensamiento crítico. ${ }^{9}$

DePaola acertadamente escribe sobre la importancia de un cambio en el enfoque de la educación odontológica, y menciona que tanto profesores como estudiantes deben ser capaces de estar a la vanguardia en los avances científicos y tecnológicos que tengan impacto en la práctica dental. Señala que el desarrollo de microarreglos de las especies bacterianas orales puede contribuir como herramientas de diagnóstico, que el estudio de biomarcadores de la inflamación puede permitir tratamientos adecuados y que el desarrollo de la biología de las células madres y la medicina regenerativa requiere de revitalizar la enseñanza odontológica. ${ }^{10}$ Para seguir los avances científicos y tecnológicos, se requiere de una buena plataforma con saberes sólidos en disciplinas básicas, como la bioquímica, que genere fortalezas en los estudiosos de la odontología y que les permita utilizar de una forma exitosa estos conocimientos en su labor profesional.

La comprensión de la utilidad y aplicación de las áreas básicas en la educación odontológica incrementará día con día el impacto en los alumnos que cursan la carrera de cirujano dentista y pondrá de manifiesto que los cambios sociales, demográficos, científicos y tecnológicos requieren de la aplicación de estos conocimientos en la atención dental. Esto exige al futuro cirujano dentista tener una sólida preparación en estas disciplinas, por lo que la bioquímica tiene un papel trascendental en la odontología.

\section{REFERENCIAS}

1. Baum BJ. Can biomedical science be made relevant in dental education? A North American perspective. Eur J Dent Educ 2003; 7 (2): 49-55.

2. Iacopino AM. The influence of «new science» on dental education: current concepts, trends, and models for the future $J$ Dent Educ 2007; 71 (4): 450-62.

3. Brown JP. A new curriculum framework for clinical prevention and population health, with a review of clinical caries prevention teaching in U.S. and Canadian dental schools. J Dent Educ 2007; 71 (5): 572-578.

4. Haden NK, Hendricson WD, Kassebaum DK, Ranney RR, Weinstein G, Anderson EL et al. Curriculum change in dental education, 2003-09. J Dent Educ 2010; 74 (5): 539-57.

5. Spielman Al, Fulmer T, Eisenberg ES, Alfano MC. Dentistry, nursing, and medicine: a comparison of core competencies. J Dent Educ 2005; 69 (11): 1257-1271.

6. Cohen MM Jr. Major long-term factors influencing dental education in the twenty-first century. J Dent Educ 2002; 66 (3): 360373.

7. Zoller LC. Deconstruction of the curriculum: a modest proposal. J Dent Educ 2005; 69 (12): 1305-1306.

8. Zallen RD. How much basic science should be taught? The discussion continues. J Dent Educ 2006; 70 (4): 353-354.

9. De Paola's D. The importance of scientific research and teaching critical thinking in academic dental institutions: reactions to "The Revitalization of U.S. Dental Education". J Dent Educ 2008 Feb; 72(2 Suppl): 43-5.

10. DePaola DP. The revitalization of U.S. dental education. J Dent Educ 2008; 72 (2 Suppl): 28-42.

Dirección para correpondencia:

Silvia Maldonado-Frías

E-mail: biominer2007@gmail.com 